\title{
RESEARCH
}

Open Access

\section{Low-density lipoprotein receptor (LDLR) regulates NLRP3-mediated neuronal pyroptosis following cerebral ischemia/ reperfusion injury}

Rui Sun ${ }^{1,2+}$, Mengna Peng ${ }^{3 \dagger}$, Pengfei $\mathrm{Xu}^{4 \dagger}$, Feihong Huang ${ }^{5}$, Yi Xie ${ }^{3}$, Juanji $\mathrm{Li}^{3}$, Ye Hong ${ }^{3}$, Hongquan Guo ${ }^{5}$, Qian Liü ${ }^{3}$ and Wusheng Zhu ${ }^{1,3,5^{*}}$

\begin{abstract}
Background: Inflammatory response has been recognized as a pivotal pathophysiological process during cerebral ischemic stroke. NLRP3 inflammasome, involved in the regulation of inflammatory cascade, can simultaneously lead to GSDMD-executed pyroptosis in cerebral ischemia. Low-density lipoprotein receptor (LDLR), responsible for cholesterol uptake, was noted to exert potential anti-inflammatory bioactivities. Nevertheless, the role of LDLR in neuroinflammation mobilized by cerebral ischemia/reperfusion (I/R) has not been investigated.

Methods: Ischemic stroke mice model was accomplished by middle cerebral artery occlusion. Oxygen-glucose deprivation was employed after primary cortical neuron was extracted and cultured. A pharmacological inhibitor of NLRP3 (CY-09) was administered to suppress NLPR3 activation. Histological and biochemical analysis were performed to assess the neuronal death both in vitro and in vivo. In addition, neurological deficits and behavioral deterioration were evaluated in mice.

Results: The expression of LDLR was downregulated following cerebral I/R injury. Genetic knockout of $L d l r$ enhanced caspase-1-dependent cleavage of GSDMD and resulted in severe neuronal pyroptosis. LDLR deficiency contributed to excessive NLRP3-mediated maturation and release of IL-1 $\beta$ and IL-18 under in vitro and in vivo ischemic conditions. These influences ultimately led to aggravated neurological deficits and long-term cognitive dysfunction. Blockade of NLRP3 substantially retarded neuronal pyroptosis in $\mathrm{Ldll}^{-/-}$mice and cultured $\mathrm{Ldll}^{-/-}$ neuron after experimental stroke.

(Continued on next page)
\end{abstract}

\footnotetext{
* Correspondence: zwsemail@sina.com; zwsemail@sina.com

${ }^{\dagger}$ Rui Sun, Mengna Peng and Pengfei Xu contributed equally to this work.

'Department of Neurology, Jinling Clinical College of Nanjing Medical

University, 305 East Zhongshan Road, Nanjing 210002, Jiangsu Province,

China

${ }^{3}$ Department of Neurology, Jinling Hospital, Medical School of Nanjing

University, Nanjing 210002, China

Full list of author information is available at the end of the article
}

(C) The Author(s). 2020 Open Access This article is licensed under a Creative Commons Attribution 4.0 International License, which permits use, sharing, adaptation, distribution and reproduction in any medium or format, as long as you give appropriate credit to the original author(s) and the source, provide a link to the Creative Commons licence, and indicate if changes were made. The images or other third party material in this article are included in the article's Creative Commons licence, unless indicated otherwise in a credit line to the material. If material is not included in the article's Creative Commons licence and your intended use is not permitted by statutory regulation or exceeds the permitted use, you will need to obtain permission directly from the copyright holder. To view a copy of this licence, visit http://creativecommons.org/licenses/by/4.0/ The Creative Commons Public Domain Dedication waiver (http://creativecommons.org/publicdomain/zero/1.0/) applies to the data made available in this article, unless otherwise stated in a credit line to the data. 
(Continued from previous page)

Conclusions: These results demonstrated that LDLR modulates NLRP3-mediated neuronal pyroptosis and neuroinflammation following ischemic stroke. Our findings characterize a novel role for LDLR as a potential therapeutic target in neuroinflammatory responses to acute cerebral ischemic injury.

Keywords: Low-density lipoprotein receptor (LDLR), Inflammasome, Pyroptosis, Neuroinflammation, Ischemia/ reperfusion

\section{Highlights}

1. LDLR expression is downregulated following ischemic stroke.

2. Ldlr knockout facilitates cerebral infarct volume enlargement and aggravates neurological deficits.

3. LDLR could suppress neuronal pyroptosis by inhibiting NLRP3 inflammasome activation.

4. Inhibition of NLRP3 protects against pyroptotic neuronal death in $\mathrm{Ldlr}^{-1-}$ mice after ischemia.

\section{Introduction}

Ischemic stroke is a common vascular disease caused by an abrupt reduction or obstruction of cerebral blood flow [1-3]. It is one of the well-known leading causes of global death and permanent disability [4]. Post-ischemic sterile inflammation has both protective and deleterious effects on disease progression [5], among which the molecular mechanisms of neuronal inflammatory injury following cerebral ischemia are complex and remain to be fully understood.

In the central nervous system (CNS), acute cerebral ischemia/reperfusion (I/R) can trigger neuroinflammation as an essential pathophysiological process to activate the innate immune response and then a series of inflammatory cascades [6]. The initiation of the neuronal immune response involves the assembly of the inflammasome complexes. Inflammasomes are cytosolic multiprotein signaling platforms that consist of sensors such as pattern recognition receptors (PPRs) that defend against infection and damage signals [7]. Inflammasome assembly recruits pro-caspase- 1 and then process it into the mature form as caspase-1 [8]. Active caspase- 1 subsequently mediates the cleavage of gasdermin D (GSDMD) [9]. The cleavage of GSDMD releases an N-terminal fragment to bind to phosphatidylserine and cardiolipin on the plasma membrane and then exhibits the pore-forming activity, eliciting a form of lytic cell death known as pyroptosis [10, 11]. Pyroptosis is a type of programmed cell death characterized by DNA fragmentation, rapid plasma membrane rupture, cell swelling, and release of proinflammatory cellular contents $[12,13]$. It has been shown that pyroptosis could be triggered by ischemic stroke and that GSDMD could serve as a key executioner of caspase-1-mediated pyroptosis during cerebral I/R injury [10].
Low-density lipoprotein receptor (LDLR), a membranespanning glycoprotein mediating the transport and metabolism of cholesterol-containing lipoprotein, is broadly expressed on multiple cell types in various tissues [14]. In the CNS, LDLR is mainly located on the neurons, astrocytes, and oligodendrocytes [15]. LDLR plays an important role in regulating the homeostasis of the bloodstream and intracellular cholesterol [16]. Notably, except for those canonical functions, it has been reported that an increase in LDLR expression participated in restricting the deleterious proinflammatory signals of pathogens and improving prognosis among patients in sepsis and septic shock [17]. However, the precise role of LDLR in the neuronal inflammatory response following cerebral $I / R$ injury remains largely unknown.

Thus, in this study, using mouse middle cerebral artery occlusion (MCAO) model and neuronal oxygen-glucose deprivation (OGD) model, we aimed to investigate the cellular location and temporal expression of LDLR and determine whether LDLR could orchestrate post-stroke neuronal inflammatory response and pyroptosis.

\section{Materials and methods \\ Animals}

Wild-type (WT) male C57BL/6 mice were purchased from the Model Animal Research Centre of Nanjing University (Nanjing, China). C57BL/6 Ldlr knockout mice were purchased from the Nanjing Biomedical Research Institute of Nanjing University (certificate no. T001464). Mice were housed in a temperature- and humidity-controlled environment (temperature $23 \pm$ $2{ }^{\circ} \mathrm{C}$; humidity $55-60 \%$ ) with a 12 -h light $/ 12$-h dark cycle and free access to chow and water. All experimental protocols were performed in accordance with the National Institutes of Health "Guide for the Care and Use of Laboratory Animals" (NIH Publications no. 8023, revised 2011) and approved by the Experimental Animal Ethics Committee of Jinling Hospital. At least three animals were analyzed for each data point.

\section{Focal cerebral ischemia}

A total of 180 male C57BL/6 mice weighing $20-25 \mathrm{~g}$ (812 weeks old) were used in this study. The focal cerebral ischemia was accomplished by the endovascular MCAO model according to previous methods [7, 18]. In brief, 
animals were anesthetized with $2 \%$ isoflurane in $\mathrm{O}_{2}$, and the right common carotid artery, external carotid artery (ECA), and internal carotid artery (ICA) were carefully isolated through a midline cervical incision. Next, a silicon-coated monofilament (diameter $0.16 \pm 0.02 \mathrm{~mm}$ ) was inserted through the ECA and advanced into the ICA to occlude the origin of the middle cerebral artery (MCA). After ischemia for $90 \mathrm{~min}$, the suture was withdrawn to allow reperfusion. The sham operation was conducted with the same procedure, except for MCA occlusion. The body temperature of the mice was maintained at $37.0^{\circ} \mathrm{C} \pm 0.5^{\circ} \mathrm{C}$ by a heating pad until the skin incision was sutured. Altogether, $20-30 \%$ of animals were excluded because of hemorrhage, death, or failure to cause focal ischemia during surgery or after surgery.

\section{Oxygen-glucose deprivation and reoxygenation (OGD/R)}

The primary cortical neurons were extracted from the cortex of C57BL/6 mouse embryos (E14) as previously described [19]. The meninges and blood vessels were removed, followed by digestion with $0.125 \%$ trypsin for 15 min. The cells were suspended in DMEM/10\% fetal bovine serum and then seeded into flasks. After cell adherence, the medium was substituted with fresh neurobasal medium mixed with $2 \%$ B27 and 1\% glutamax, and the medium was changed every three days. The cells were cultured for 5-6 days before the OGD experiment.

For OGD, neurons were transferred to glucose-free and serum-free DMEM and then incubated in an oxygen-free chamber aerated with $5 \% \mathrm{CO}_{2}$ and $95 \% \mathrm{~N}_{2}$ at $37^{\circ} \mathrm{C}$. After $2 \mathrm{~h}$, the neurons were returned to normal culture conditions for reoxygenation.

\section{Drug administration}

A single dose of NLRP3 inhibitor CY-09 $\left(40 \mathrm{mg} \cdot \mathrm{kg}^{-1}\right.$, i.p., Selleckchem, USA) was administered $1 \mathrm{~h}$ before MCAO surgery as described previously [20]. In cultured neurons, CY-09 $(10 \mu \mathrm{M})$ was pre-incubated for $0.5 \mathrm{~h}$ before OGD. Then, neurons were incubated with CY-09 $(10 \mu \mathrm{M})$ for $24 \mathrm{~h}$ after reoxygenation. Vehicle animals were treated with equivalent normal saline (i.p.) for comparison.

\section{Cerebral infarct volume and cerebral water content}

At $24 \mathrm{~h}$ after reperfusion, the mice were anesthetized, and their brains were quickly removed. Then, 2,3,5-triphenyltetrazolium chloride (TTC, Sigma) staining was employed to measure brain infarct volume. Mouse brains were cut into $1-\mathrm{mm}$ sections and stained with TTC solution for $15 \mathrm{~min}$ at $37^{\circ} \mathrm{C}$ before being fixed with $4 \%$ paraformaldehyde (PFA) at $4{ }^{\circ} \mathrm{C}$ overnight. The relative infarct volume was calculated as reported previously [21]. The brain water content was measured with the wet-dry method [22]. Each hemisphere was weighed after removal and then weighed again at $105^{\circ} \mathrm{C}$ overnight. The percentage of water content was calculated as [(wet weight - dry weight)/wet weight] $\times 100 \%$.

Evaluation of neurological deficits and behavioral analysis Neurological deficits of the experimental mice were assessed with the modified neurologic severity score (mNSS) 24h after reperfusion, as described [23]. The mNSS scoring system consists of four tests: sensory tests, motor tests, beam balance tests, and reflexes absent and abnormal movements. The mNSS is graded a scale of $0-18$ points in which higher score indicates more severe neurological deficits. One point is recorded for the failure to perform the task or lack of a reflex. The score of 13-18 indicates severe injury while that of 1-6 indicates mild injury.

Spatial learning and memory were investigated with the Morris Water Maze (MWM) test on days 22-28 after reperfusion [24]. A blind test was performed prior to the experimental task on day 22 to exclude blind mice. In the place navigation test, animals were trained to find the platform in four trials for 5 days. Each trial lasted until the animal found the platform in $60 \mathrm{~s}$. If the animal failed to reach the platform within $60 \mathrm{~s}$, it would be guided there and rested for $10 \mathrm{~s}$ before the next experiment. In the spatial probe test on day 28 , the platform was removed, and each animal was placed to swim freely for $60 \mathrm{~s}$. The time spent in the target quadrant and the number of platform crossings were recorded and analyzed by the ANY-maze video tracking software (Stoelting, USA).

\section{Cell viability}

Twenty-four hours after OGD, neuronal cell viability was detected with the Cell Counting Kit-8 (CCK-8) (Dojindo, Japan) according to the manufacturer's instructions. The absorbance of each well was obtained at $450 \mathrm{~nm}$. Cell viability was calculated by (experimental group absorbance value/control group absorbance value) $\times 100 \%$.

Meanwhile, cell death was determined with a propidium iodide (PI)/Hoechst 33342 assay kit (Thermo Fisher Scientific, USA). The percentage of propidium iodide-positive neurons (red) compared with the total Hoechst-stained neurons (blue) was used to assess cell death. For each sample, four randomly selected areas were counted, and the average value was calculated.

\section{Fluoro-jade C (FJC) staining}

Degenerated neurons were detected by FJC (Millipore, USA) as previously described [25]. Frozen slides were sequentially immersed in $1 \%$ sodium hydroxide solution, $70 \%$ ethanol, and $0.06 \%$ potassium permanganate solution. Then, the sections were then incubated with 
$0.0001 \%$ solution of FJC. Quantified analysis of FJCpositive neurons was performed with Image J software.

\section{Immunofluorescence}

Anesthetized mice were successively perfused intracardially with $0.9 \%$ sodium chloride and $4 \%$ paraformaldehyde (PFA). Mouse brains were soaked in $4 \%$ PFA for $12 \mathrm{~h}$ and then dehydrated by sucrose solutions with an ascending concentration gradient of 10 , 20 , and $30 \%$ at $4{ }^{\circ} \mathrm{C}$. After fixation in optimal cutting temperature compound (Sakura Finetek, USA), the brains were sliced into $15-\mu \mathrm{m}$ sections. Brain sections and neuron coverslips were fixed with 4\% PFA for 20 min and then permeabilized with blocking buffer comprising $5 \%$ goat serum, $1 \%$ bovine serum albumin (BSA) and $0.3 \%$ Triton $\mathrm{X}-100$ at room temperature for $1 \mathrm{~h}$. The samples were incubated overnight with primary antibodies against LDLR (1:200, Santa Cruz Biotechnology, USA), NeuN (1:500, Abcam, UK), GFAP (1:500, Abcam, UK), Iba-1 (1:100, Abcam, UK), NLRP3 (1:200, Abcam, UK), ASC (1:200, Santa Cruz Biotechnology, USA), Caspase-1 (1:200, Santa Cruz Biotechnology, USA), and Gasdermin D (1:200, Santa Cruz Biotechnology, USA) at $4{ }^{\circ} \mathrm{C}$, followed by incubation with appropriate Alexa Fluor-488/594-conjugated secondary antibodies (Jackson ImmunoResearch) and DAPI. Immunofluorescence images were captured with a microscope (Olympus MX51, Japan). The positive signals were analyzed using ImageJ software.

\section{Real-time quantitative PCR}

Total RNA was extracted from cerebral tissues or cultured neurons utilizing TRIzol Reagent (Sigma, USA), and cDNA was reverse transcribed with a PrimeScript RT reagent kit (Thermo Fisher Scientific, USA). Realtime PCR was implemented in a Stratagene Mx3000P QPCR system (Agilent Technologies, USA) using a reaction system (UItraSYBR Mixture (ComWin Biotech, China), specific primers, diluted cDNA). The levels of glyceraldehyde-3-phosphate dehydrogenase (GAPDH) were set as the internal reference to assess the expression of target genes. The primer pairs are listed in Table 1.

\section{ELISA assay for inflammatory cytokines}

IL-1 $\beta$ and IL-18 in cerebral ischemic penumbra and neuron culture supernatants were detected and quantified by ELISA kits (Abcam, UK) following the manufacturer's instructions. Briefly, the supernatant of brain tissue homogenate or neuronal medium was added to 96-well plates coated with the indicated antibodies. After the reaction between the enzyme and substrate, the absorbances of the sample were assessed at $450 \mathrm{~nm}$ using a microplate reader (Thermo Fisher Scientific, USA).
Table 1 Real-time PCR primers used in this study

\begin{tabular}{lll}
\hline Primer name & \multicolumn{2}{l}{ Primer sequence } \\
\hline NLRP3 & Forward & ATGCTGCTTCGACATCTCCT \\
& Reverse & AACCAATGCGAGATCCTGAC \\
Pro-IL-1 & Forward & CAGGCAGGCAGTATCACTCA \\
& Reverse & AGGCCACAGGTATTTTGTCG \\
Pro-IL-18 & Forward & GACTCTTGCGTCAACTTCAAGG \\
& Reverse & CAGGCTGTCTITTGTCAACGA \\
GAPDH & Forward & AAGAAGGTGGTAAGCAGGC \\
& Reverse & TCCACCACCCAGTTGCTGTA \\
\hline
\end{tabular}

\section{Electron microscopy}

Tissues $(1 \times 1 \times 1 \mathrm{~mm})$ dissected from the cerebral ischemic penumbra were successively fixed in $2.5 \%$ glutaraldehyde and $1 \%$ osmium tetroxide and then cut into $50-60 \mathrm{~nm}$ slices after dehydration and insertion. Samples were observed and scanned using an H7500 Transmission Electron Microscope (Hitachi, Japan).

\section{CHOD-PAP method}

Cholesterol levels in fresh venous serum and various brain regions were abstracted and detected using a Cholesterol Kit (Biosino Bio-technology, China) following the manufacturer's instructions.

\section{Immunoblotting analysis}

Immunoblotting analysis was performed as reported previously $[19,26]$. Protein samples from brain tissues and cultured neurons were extracted using RIPA lysis buffer (Cell Signaling Technology, USA). The concentrations of these samples were detected with a BCA assay (Generay Biotechnology, China). Protein samples $(20 \mu \mathrm{g}$ for cells, $30 \mu \mathrm{g}$ for tissues) were subjected to $8-12 \%$ sodium dodecyl sulfate-polyacrylamide gel electrophoresis (SDSPAGE) and then transferred to polyvinylidene difluoride (PVDF) membranes (Millipore, USA). The membranes were probed overnight at $4{ }^{\circ} \mathrm{C}$ with primary antibodies against LDLR (1:200, Abcam, UK), NLRP3 (1:500, Abcam, UK), ASC (1:500, Santa Cruz Biotechnology, USA), Caspase-1 p10 (1:500, Santa Cruz Biotechnology, USA), GSDMD (1:500, Santa Cruz Biotechnology, USA), IL-1 $\beta$ (1:500, Santa Cruz Biotechnology, USA), IL-18 (1:500, Abcam, UK), nuclear factor kappa B (NF-кB) p65 (1:1000, Cell Signaling Technology, USA), phosphorylated-NF- $\mathrm{kB}$ p65 (1:1000; Abcam, UK), and $\beta$-actin (1:3000, Cell Signaling Technology, USA). The membranes were then incubated with horseradish peroxidase (HRP)-conjugated secondary antibody for $1 \mathrm{~h}$ at room temperature. Protein signals were detected by enhanced chemiluminescence solution (ECL, Millipore, USA). Quantitative analysis of protein bands was conducted using ImageJ software. $\beta$-actin served as the internal control. 


\section{Statistical analysis}

Experimental data were analyzed by SPSS 22.0 software (SPSS, Chicago, IL, USA). All values are expressed as the mean \pm SEM. Escape latency and swimming path length in the MWM test were analyzed by two-way repeatedmeasures ANOVA followed by the least-significantdifference (LSD) post hoc test to determine differences between groups. Other results were analyzed using an independent sample $t$ test for comparing two groups and one-way ANOVA for comparing multiple groups followed by LSD post hoc test. Statistical significance was taken as $P<0.05$.

\section{Results}

The expression of neuronal LDLR is decreased following cerebral I/R

Immunofluorescence results indicated that LDLR was mainly detected in the cortex and corpus callosum of mice, with relatively fewer present in the hippocampus (Fig. 1a, S1A, S1B). In the cerebral cortex, LDLR signals were chiefly observed in the membrane and cytoplasm of neurons, rather than in the GFAP-positive astrocytes or Iba1-positive microglia (Fig. 1a-c). These results indicated that LDLR was mainly expressed in neurons located in the cortical area.

In MCAO mice, we observed weaker immunostaining intensity of neuronal LDLR in the peri-infarct regions than in sham-operated mice (Fig. 1a). Next, we assessed the temporal profile of LDLR protein expression in ischemic penumbra post-stroke. The western blotting results indicated that LDLR level was decreased after reperfusion and showed a robust decline at $24 \mathrm{~h}$ (Fig. 1d, compared to sham $12 \mathrm{~h}$ group, $P<0.001$ for reperfusion $12 \mathrm{~h} ; P<0.001$ for reperfusion $24 \mathrm{~h} ; P<0.001$ for reperfusion $72 \mathrm{~h}$ ). Furthermore, the expression of LDLR in cultured neurons also declined $24 \mathrm{~h}$ after reoxygenation (Fig. 1e, compared to the control $24 \mathrm{~h}$ group, $P<0.001$ for reoxygenation 24 h; $P=0.002$ for reoxygenation $72 \mathrm{~h}$ ).

\section{Ldlr knockout aggravates post-stroke neurological deficits, infarct progression, and brain edema}

We further utilized $L d l r$ knockout mice to examine the physical function of LDLR in ischemic stroke. Western blotting analysis confirmed the complete knockout of LDLR protein in $\mathrm{Ldlr}^{-1-}$ mice (Fig. 2a, compared to WT sham group, $P<0.001$ for $L d l r^{--}$sham group). Due to the significant change of LDLR expression at $24 \mathrm{~h}$ after reperfusion, we chose $24 \mathrm{~h}$ as the detecting timepoint after MCAO treatment.

The mNSS assessment showed that MCAO surgery significantly destroyed sensorimotor function, as the neurological deficit score climbed to $7.67 \pm 0.29$ (Fig. 2b, compared to WT sham group, $P<0.001)$. In MCAOtreated $\mathrm{Ldll}^{-/-}$mice, neurological function was further exacerbated with a score of $9.73 \pm 0.51$ (Fig. 2b, compared to the WT MCAO group, $P<0.001)$. Regarding the brain water content, a similar tendency was observed in $\mathrm{Ldlr}^{-1-}$ MCAO mice where it changed from $80.89 \% \pm$ $1.55 \%$ to $86.88 \% \pm 2.82 \%$ (Fig. 2c, compared to WT MCAO group, $P=0.028$ ). As demonstrated in Fig. $2 \mathrm{~d}$ and e, the percentage of infarct volume was markedly enlarged to $37.34 \% \pm 2.96 \%$ by genetic ablation of $L d l r$ following MCAO, as detected by TTC staining (compared to WT MCAO group, $P=0.001$ ). We then used FJC staining to test whether LDLR could affect the neuronal degeneration after ischemic stroke. The images displayed that FJC-positive neurons after MCAO were significantly increased by inhibition of LDLR (Fig. 2f, compared to WT MCAO group, $\mathrm{p}<0.001)$. These results suggested that $L d l r$ knockout aggravates early brain injury after ischemic stroke.

\section{Ldlr knockout exacerbates long-term cognitive deterioration}

To detect long-term spatial learning and memory function, we next performed MWM tests. The neurobehavioral capacities of $\mathrm{Ldlr}^{-/-}$and their WT littermates both deteriorated in response to cerebral I/R, and compared with WT MCAO mice, $\mathrm{Ldlr}^{-1-}$ MCAO mice swam a longer distance and took more time to reach the platform on the 5th training day (Fig. 3a-c, compared with WT MCAO group, $P=0.045$ for path length; $P=0.028$ for escape latency). In the probe phase, no significant differences were observed in the WT sham and $L d l r^{-1}$ sham groups. Both the time spent in the target quadrant and platform crossovers of WT MCAO mice were identically inhibited in the place navigation test (Fig. 3d, e, compared with WT sham group, $P=0.012$ ). Simultaneously, LDLR deficiency conspicuously decreased the time spent in the target quadrant and platform crossovers (Fig. 3d, e, compared with the WT MCAO group, $P=0.046$ and $P=0.043$, respectively). Taken together, the loss of LDLR may have substantial detrimental effects on long-term behavioral outcomes following cerebral ischemic stroke.

\section{LDLR deficiency promotes neuronal pyroptosis post- stroke}

Previous studies have identified cerebral I/R-induced fierce cell pyroptosis in the ipsilateral ischemic hemisphere, where GSDMD may serve as a pivotal executioner $[7,10]$. Therefore, we subsequently sought to determine whether LDLR could affect neuronal pyroptosis following ischemia. The western blot results showed that the expression levels of the full-length and $\mathrm{N}$ terminal parts of GSDMD were both elevated at $12 \mathrm{~h}$ after reperfusion and reached a peak at $24 \mathrm{~h}$ after reperfusion, which remained at levels higher than 


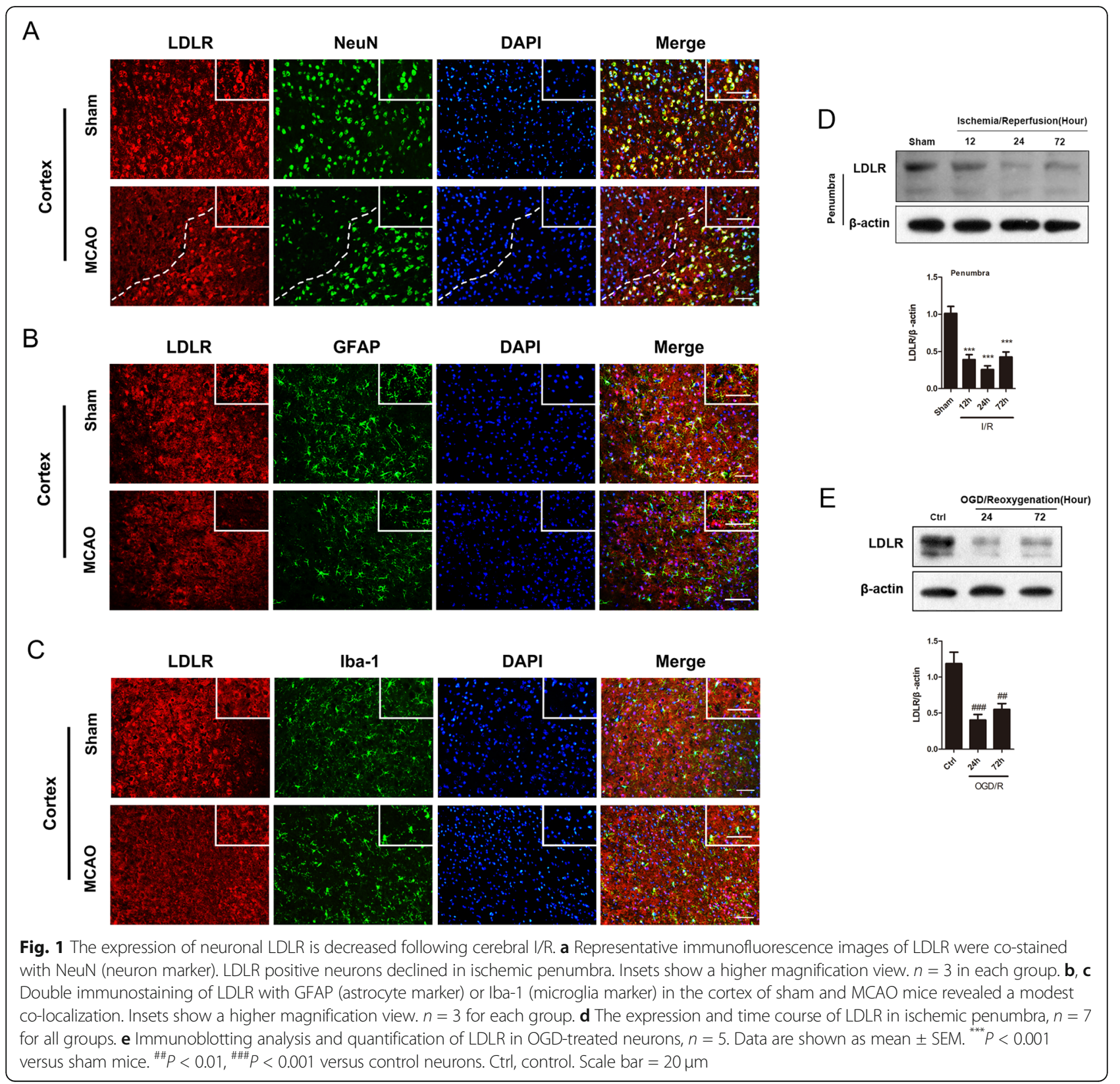

baseline at $72 \mathrm{~h}$ after reperfusion (Fig. 4a, compared with Sham group, full-length GSDMD: $P=0.005$ for reperfusion $12 \mathrm{~h}, P<0.001$ for reperfusion $24 \mathrm{~h}, P=$ 0.017 for reperfusion $72 \mathrm{~h}$; N-terminal GSDMD: $P<$ 0.001 for reperfusion $24 \mathrm{~h}$ ). Cerebral $\mathrm{I} / \mathrm{R}$ attack increased the expression of full-length GSDMD and Nterminal GSDMD to 3.3-fold and 3.2-fold higher than those in the sham group, which were both boosted by LDLR deletion (Fig. 4b, compared with the WT MCAO group, $P=0.006$ for full-length GSDMD; $P<$ 0.001 for $\mathrm{N}$-terminal GSDMD). Immunostaining manifested that GSDMD-positive neurons were markedly increased in the ischemic penumbra region $24 \mathrm{~h}$ after MCAO, and the trends were notably enhanced by Ldlr knockout (Fig. 4c, d, compared with shamoperated group, $P<0.001$ for WT MCAO group; compared with WT MCAO mice, $P<0.001$ for the $\mathrm{Ldlr}^{-1-}$ MCAO group). Meanwhile, the secretion of IL-1 $\beta$ and IL-18 in ischemic penumbra was upregulated to $112.62 \pm 12.67 \mathrm{pg} / \mathrm{ml}$ and $524.56 \pm 83.96 \mathrm{pg} /$ $\mathrm{ml}$, which was further aggravated to $152.85 \pm 18.66$ $\mathrm{pg} / \mathrm{ml}$ and $776.95 \pm 124.74 \mathrm{pg} / \mathrm{ml}$ by LDLR deficiency (Fig. 4e, f, compared with WT MCAO group, $P=$ 0.047 for IL-1 $\beta, P=0.041$ for IL-18). In addition, 


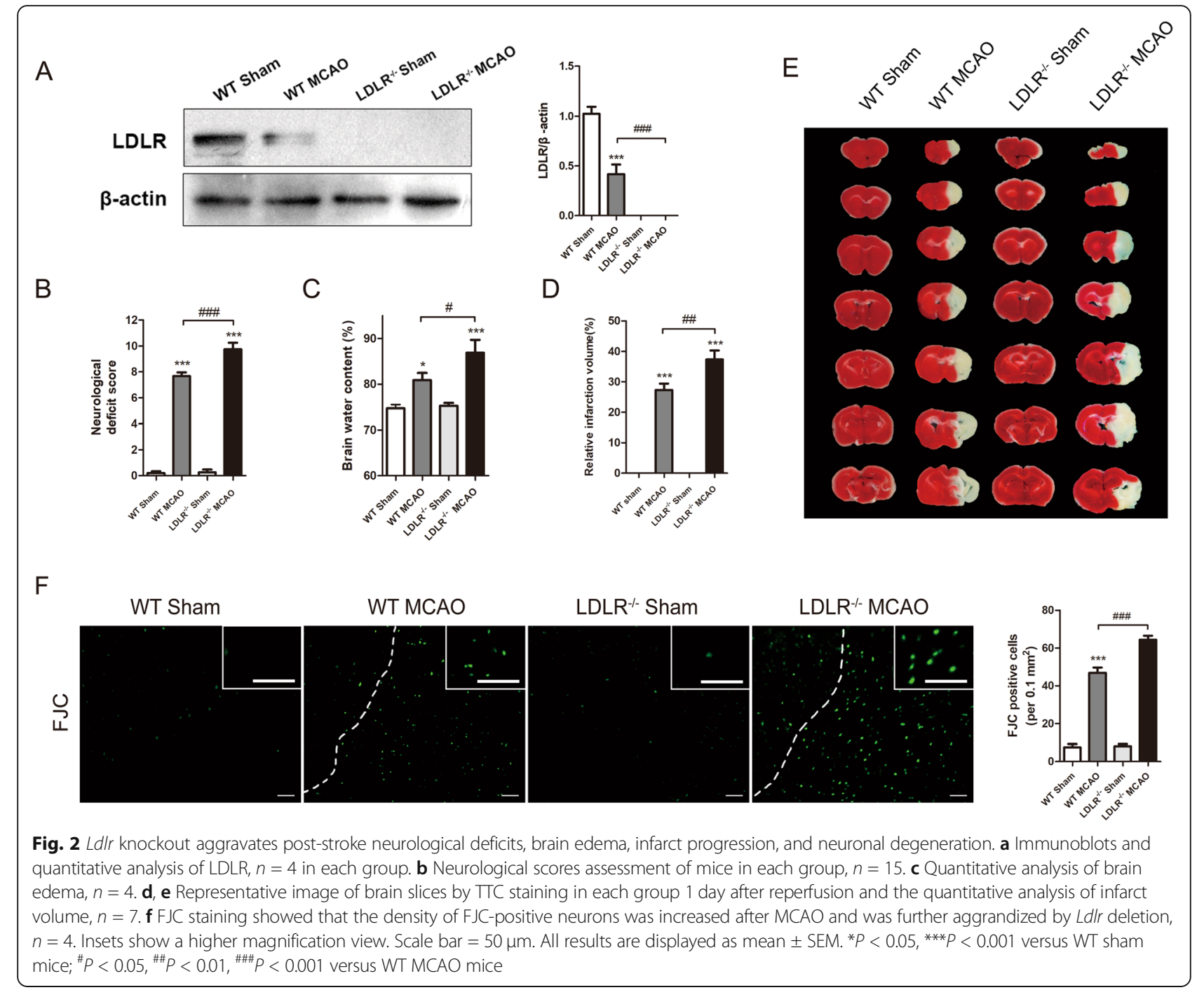

TEM images showed that more GSDMD pores were exhibited on neurons in $\mathrm{Ldlr}^{-1-} \mathrm{MCAO}$ mice compared with those in WT MCAO mice (Fig. 4g).

To investigate the specific role of LDLR in neuronal pyroptosis, we cultured primary cortical neurons and performed OGD treatment. Neuronal death was provoked $24 \mathrm{~h}$ after OGD/R administration and was further magnified by LDLR elimination, as detected by PI/Hoechst staining (Fig. 5a, b, compared with WT OGD group, $P=0.013)$. Likewise, cell viability declined considerably in $\mathrm{Ldlr}^{-1-}$ neurons compared to that of WT neurons (Fig. $5 \mathrm{c}, P=0.046$ ). Immunofluorescence staining revealed that LDLR deletion further increased the number of GSDMD-positive neurons (Fig. 5d). The protein level of full-length GSDMD was augmented by $\mathrm{OGD} / \mathrm{R}$ administration (Fig. 5e, compared with WT Ctrl group, $P=0.049$ for WT OGD group) and more prominently under
LDLR deficiency (Fig. 5e, compared with the WT OGD group, $P=0.04$ for the $L d l r^{-/-}$OGD group). An analogous tendency was found for the level of the $\mathrm{N}$-terminal GSDMD in neurons, which was the key biological marker of pyroptosis (Fig. 5e, compared to WT OGD group, $P=0.021$ ). As shown in Fig. $5 \mathrm{f}$ and $\mathrm{g}$, the reoxygenation-induced release of IL-1 $\beta$ and IL18 was also elevated in the culture medium of $\mathrm{Ldll}^{-/-}$ OGD neurons (compared to WT OGD group, $P=$ 0.041 for IL-1 $\beta ; P=0.048$ for IL-18).

Ldlr deletion amplifies NLRP3 inflammasome activation in cerebral I/R injury

With normal chow, plasma cholesterol levels were only 1.1-fold higher in $\mathrm{Ldll}^{-/-}$mice than levels in WT mice (Fig. S2A, $P<0.001$ ). Since excessive cholesterol may affect sterile inflammation following 


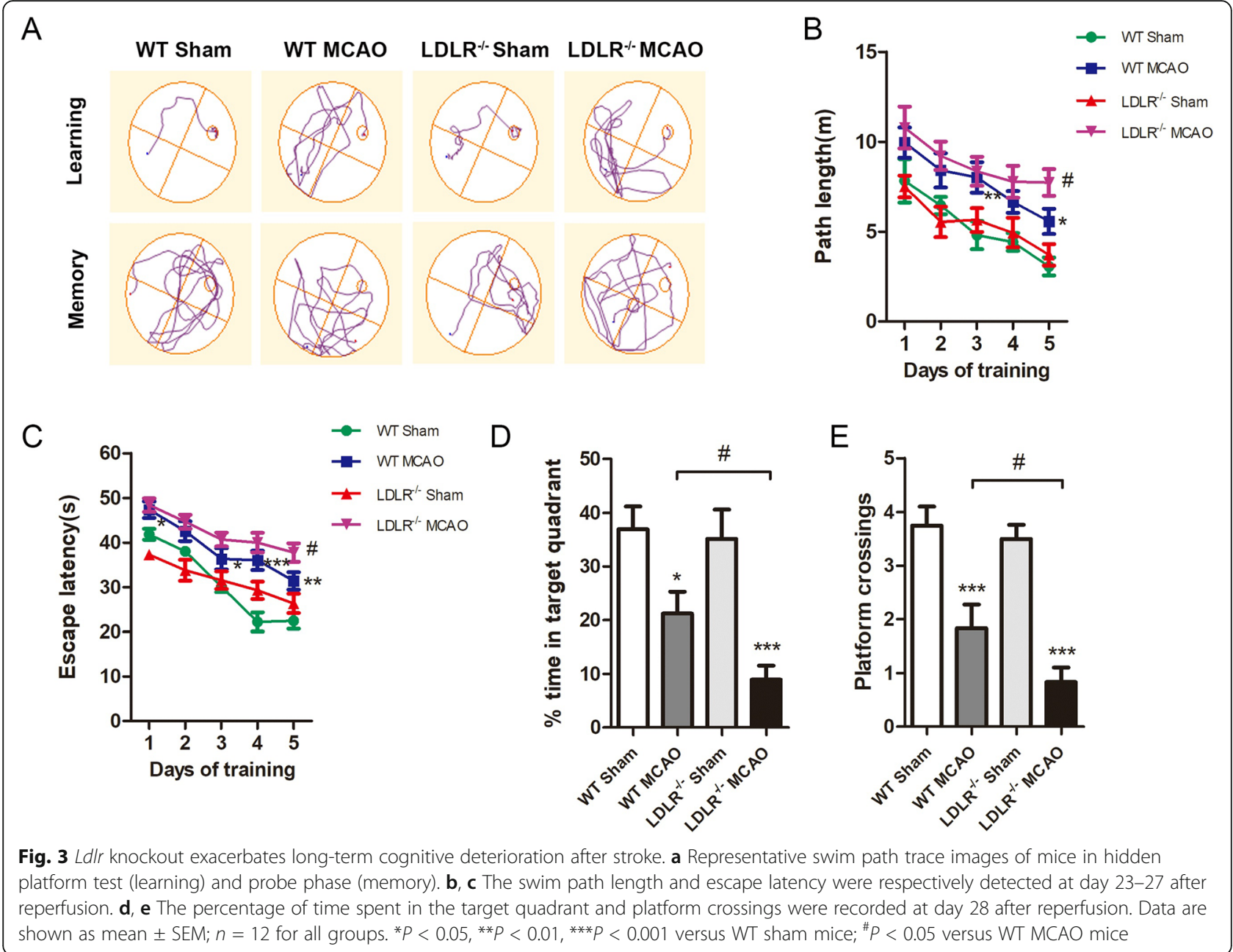

ischemic stroke, tissue cholesterol levels were measured in different regions of the mouse brain. No significant differences were noted among the groups (Fig. S2B).

The canonical pathway of pyroptosis is mediated by inflammasome activation following ischemic stroke, which also processes the precursors of IL-1 $\beta / 18$ into their mature forms and triggers neuroinflammation [27]. Given that the activation of the NLRP3 inflammasome requires an initial priming signal to evoke NF- $K B$ signaling [2], we next asked whether LDLR could affect inflammasome activation and whether the NF- $\mathrm{KB}$ pathway was involved. Double staining results showed that ischemia-induced elevations of NLRP3, ASC, and caspase- 1 were prominently augmented by LDLR deletion in peri-infarct regions (Fig. $6 \mathrm{a}-\mathrm{d}$, compared to WT MCAO group, $P=0.048$ for NLRP3; $P$ $=0.012$ for ASC; $P<0.001$ for caspase-1). Enhanced protein expression of NLRP3, ASC, cleaved caspase-1, mature IL-1 $\beta$, and IL-18 was detected in $\mathrm{Ldlr}^{-/-}$mice after $\mathrm{I} / \mathrm{R}$ insult (Fig. 6e, f, compared to WT MCAO group, $P=0.001,0.002,0.001,0.048$, and 0.01 , respectively). No significant differences were observed in the expression of caspase-1 precursor and pro-IL$1 \beta$ (Fig. 6e, f).

Consistently, as exhibited in Fig. 7a, in vitro OGD/R strengthened the immunofluorescence intensity of NLRP3, ASC, and caspase-1 in primary cultured neurons, which was further enhanced in $\mathrm{Ldlr}^{-1-}$ neurons. Deficiency of LDLR led to marked increases in the protein expression of NLRP3, cleaved caspase-1, and IL-18 in OGD/R neurons (Fig. 7b, c, e, f, compared with the WT OGD group, $P=0.016,0.024$, and 0.047 , respectively). No significant difference was observed in the expression of caspase- 1 precursor in vitro (Fig. $7 \mathrm{~b}, \mathrm{~d}$ ).

Thereafter, the role of NF-kB pathway in NLRP3 inflammasome activation was detected. The protein levels of phosphorylated-p65 (p-p65) were upregulated by 3.6-fold in the penumbra regions of MCAO mice (Fig. 8a, compared to WT sham group, $P=0.019$ ), while $L d l r$ knockout substantially increased their expression to 5.76-fold, respectively (Fig. 8a, compared 


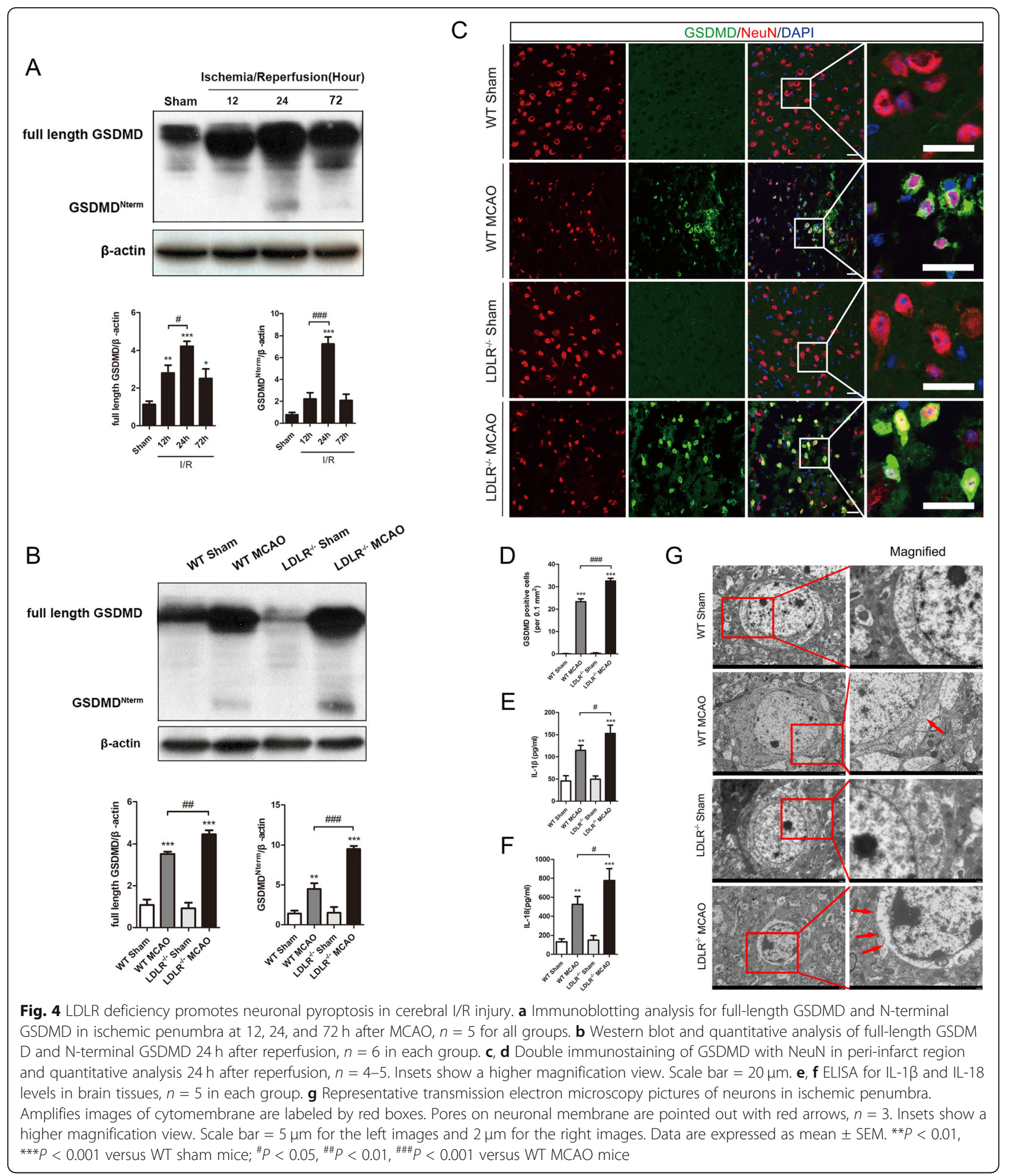

to WT MCAO group, $P=0.043)$. Similar elevations of p-p65 expression were verified in cultured $L d l r^{-/-}$ neurons treated with OGD/R (Fig. 8b, compared to WT OGD group, $P=0.001$ ). No significant difference was detected in the expression of p65 both in vivo and in vitro. A striking increase in pro-IL-1 $\beta$, pro-IL18, and NLRP3 mRNAs was confirmed in $L d l r^{-/}$ mice following I/R examined by real-time PCR (Fig. $8 \mathrm{c}$, compared with WT MCAO group, $P=0.038$, 0.022 , and 0.009 , respectively). The mRNA levels of 


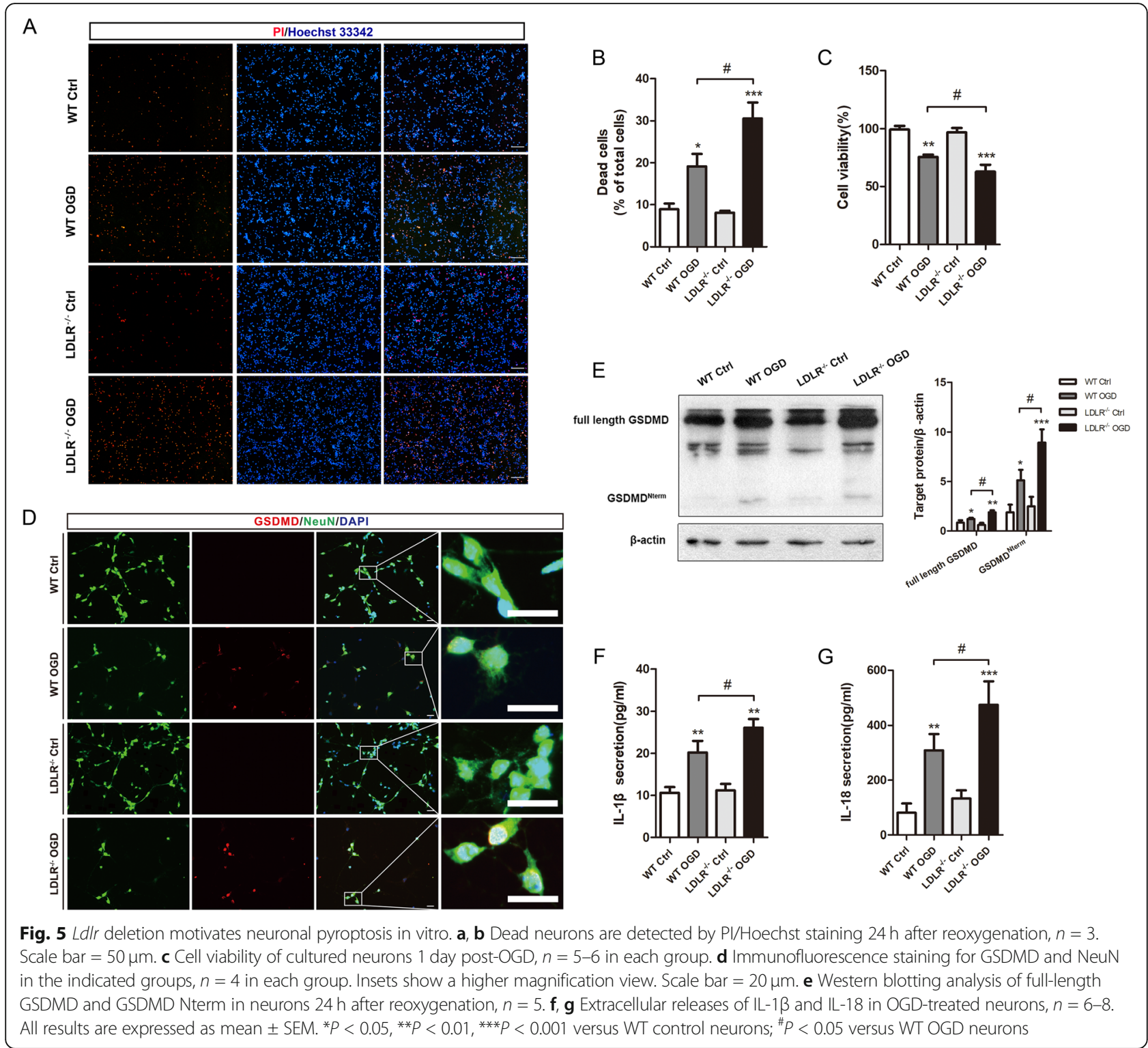

pro-IL-1 $\beta$, pro-IL-18, and NLRP3 were also increased in $\mathrm{Ldll}^{-1-}$ neurons after reoxygenation (Fig. 8d, compared to WT OGD neurons, $P=0.038,0.007$, and 0.007 , respectively).

\section{Inhibition of NLRP3 reverses Ldlr deficiency-induced augment of neuronal pyroptosis following ischemia}

To investigate whether LDLR regulated neuronal pyroptosis and inflammatory response via mediating NLRP3 inflammasome, we treated $\mathrm{Ldlr}^{-/-}$mice or $\mathrm{Ldlr}^{-1-}$ neurons with CY-09 to inhibit NLRP3. As shown in Fig. 9a and b, ischemia-triggered elevation of NLRP3 expression was conspicuously restrained with the administration of CY-09 (compared to $\mathrm{Ldlr}^{-1-}$ MCAO group, $\left.P<0.001\right)$. The noticeable increment of ASC, cleaved caspase-1, mature IL-1 $\beta$, and
IL-18 levels in $\mathrm{Ldlr}^{-/-}$mice were all suppressed by CY-09 (Fig. 9a, b, compared to $\mathrm{Ldlr}^{-1-}$ MCAO group, $P<0.001$ for ASC, $P=0.045$ for cleaved caspase- 1 ; $P$ $<0.001$ for IL-18; $P=0.01$ for mature IL-1 $\beta$ ). Then, we detected the expression of GSDMD after NLPR3 inhibition. Employment of CY-09 significantly retarded LDLR deficiency-induced increment of fulllength GSDMD and N-terminal GSDMD following ischemia (Fig. 9c, d, compared with $\mathrm{Ldlr}^{-1-} \mathrm{MCAO}$ group, $P=0.005$ for full-length GSDMD; $P=0.004$ for N-terminal GSDMD). We next applied CY-09 to $\mathrm{Ldlr}^{-1-}$ neurons. The elevated expression of NLRP3 induced by OGD was refrained by CY-09 (Fig. 9e, f, compared to $\mathrm{Ldlr}^{-1-}$ OGD group, $\left.P=0.012\right)$. Similar tendencies of ASC, cleave caspase-1, IL-18, IL-1 $\beta$, full-length GSDMD, and N-terminal GSDMD 


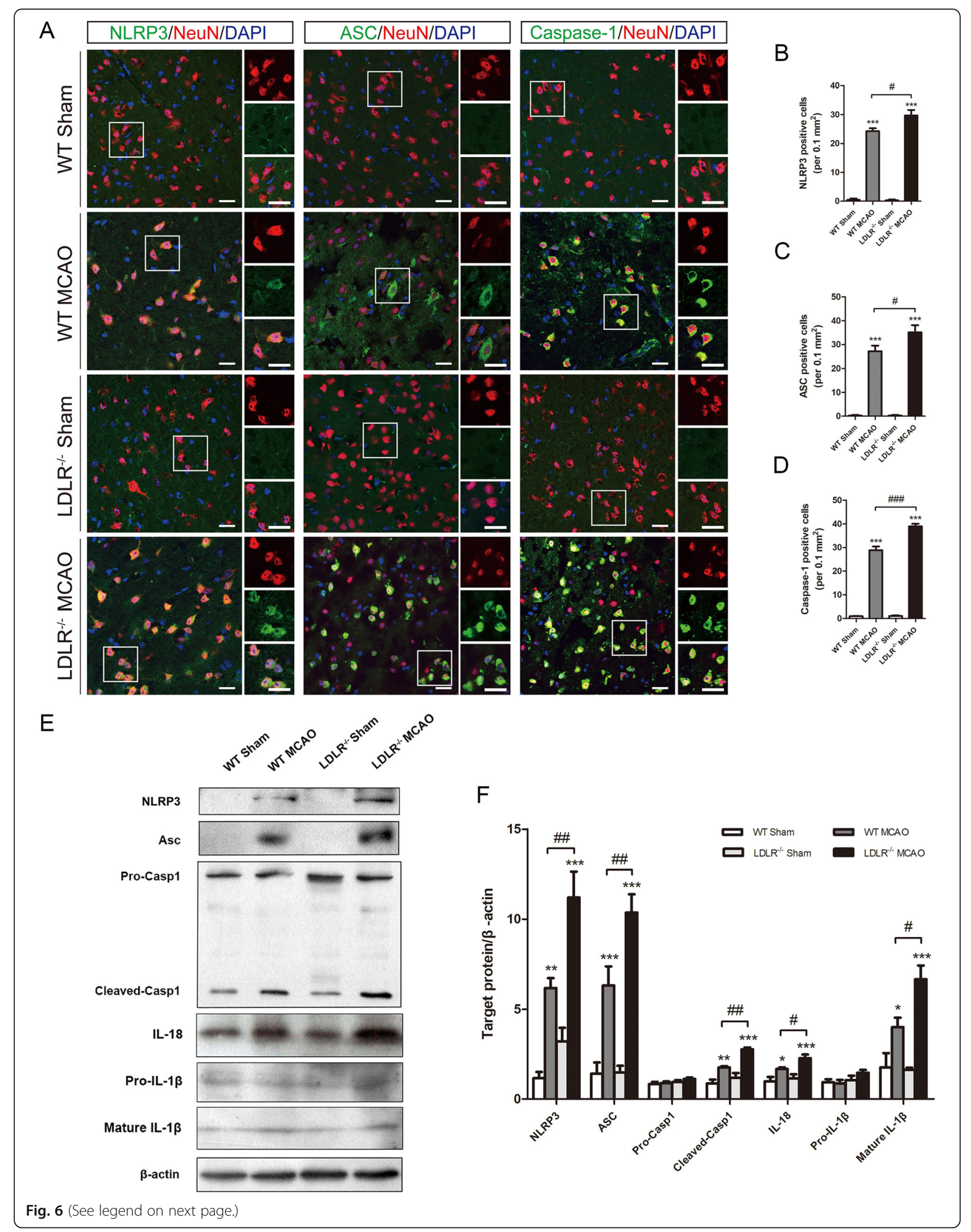


(See figure on previous page.)

Fig. 6 Ldlr knockout amplifies ischemia-induced NLRP3 inflammasome activation. a-d Representative immunofluorescence images of NLRP3, ASC, and caspase- 1 were co-stained with NeuN in peri-infarct areas and their quantification $24 \mathrm{~h}$ after reperfusion, $n=3-5$ in each group. Insets show a higher magnification view. Scale bar $=20 \mu \mathrm{m}$. e, f Western blotting and quantitative analysis of NLRP3, ASC, caspase-1, IL-18, and IL-1 $\beta$ expression in ischemic penumbra tissue $24 \mathrm{~h}$ after reperfusion, $n=4-6$ for each group. Data are indicated as mean \pm SEM. ${ }^{*} P<0.05$, ${ }^{*} P<0.01$,

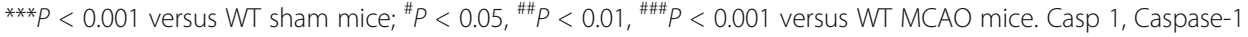

expressions were also observed in $\mathrm{Ldlr}^{-/-}$neurons treated with CY-09 after OGD/R (Fig. 9e-h, compared with $L d l r^{-/}$OGD group, $P=0.027,0.004$, $0.021,0.018,0.048,0.021$, respectively). All these results demonstrated that LDLR regulates neuronal pyroptosis and neuroinflammation via NLRP3 inflammasome pathway.

\section{Discussion}

The present study demonstrated that the expression of LDLR was downregulated following acute cerebral ischemia. Furthermore, $L d l r$ genetic knockout exacerbated neuronal pyroptosis and inflammatory response by provoking NLRP3 inflammasome activation and recruitment, leading to cerebral infarct volume enlargement and neurological deficit aggravation (Fig. 10). Inhibition of NLRP3 could reverse enhanced neuronal pyroptosis induced by $L d l r$ deficiency after ischemia. Moreover, post-stroke long-term cognitive and memory impairments of mice were deteriorated by Ldlr deletion.

Neuroinflammation in the CNS is an essential process in the pathophysiology of ischemic stroke, which can result in severe nerve injury and long-term neurobehavioral dysfunction [28]. However, the progress in seeking proper treatments aimed at reducing neuroinflammation following ischemia remains limited. Previous evidence has provided insight into a sterile inflammatory mechanism known as "inflammasome" during acute cerebral ischemia [29]. Inflammasomes are innate immunity hubs, generally composed of a stimulus-detecting sensor, the adaptor molecule ASC, and the protease precursor procaspase-1, playing a critical role in the initiation of innate immune response through sensing stimulusinduced danger-associated molecular patterns (DAMPs) released from infarct regions [30-34]. Among multiple inflammasome-forming proteins, NLRP3 has been reported to regulate neuroinflammation and neuronal

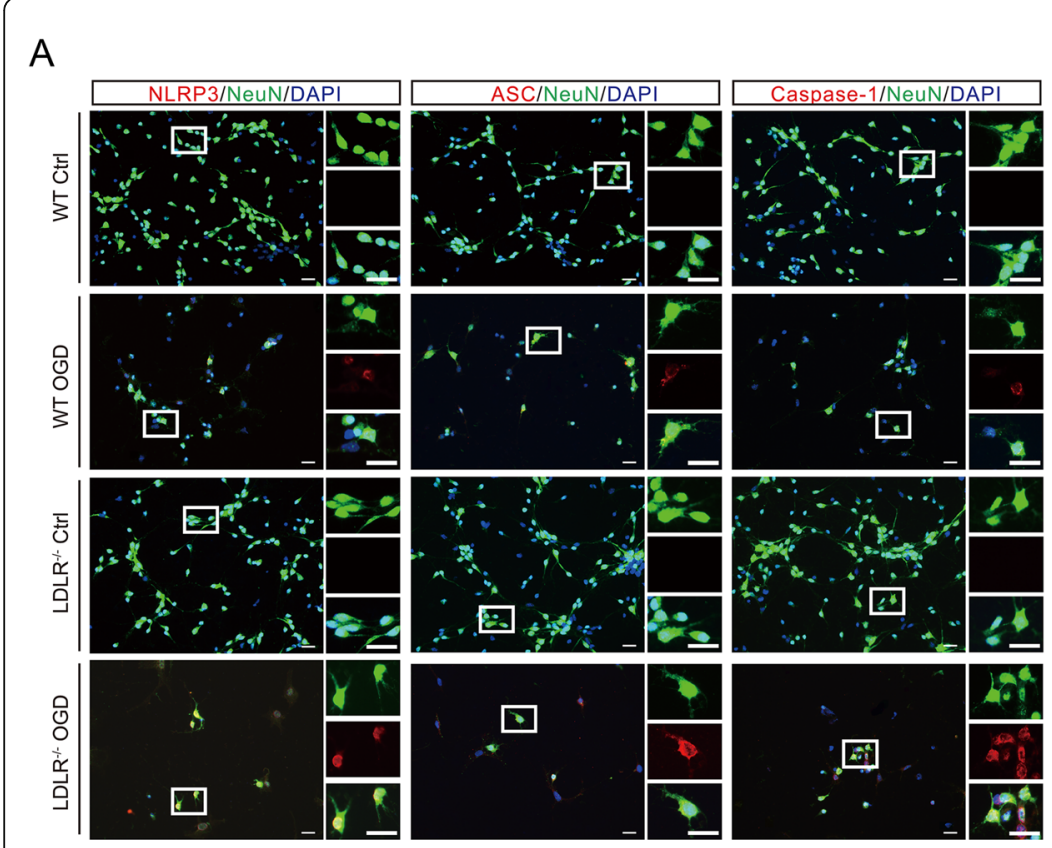

B

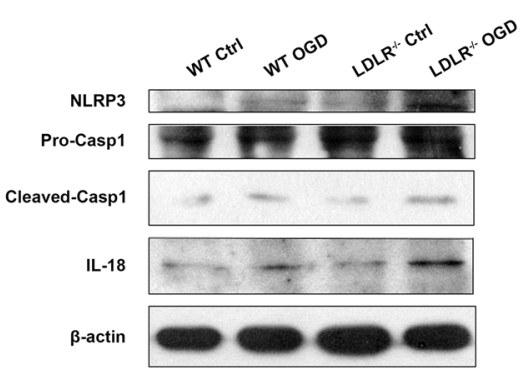

C

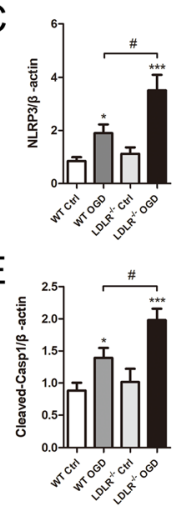

D

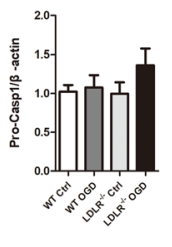

$\mathrm{F}$

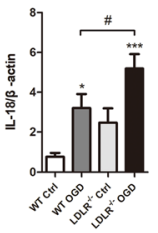

Fig. 7 Absence of LDLR fortifies neuronal inflammasome activation after OGD/R. a Representative immunofluorescence images and quantitative analysis of NLRP3, ASC, and caspase- 1 in cultured neurons $24 \mathrm{~h}$ after reoxygenation, $n=4$. Insets show a higher magnification view. Scale bar $=$ $20 \mu \mathrm{m}$. b-f Immunoblotting analysis and quantification of NLRP3, caspase-1, and IL-18 expressions in primary neurons, $n=5$ for IL-18 and $n=4$ for other proteins. All data are expressed as mean \pm SEM. ${ }^{*} P<0.05$, ${ }^{* * *} P<0.001$ versus $W T$ control neurons; ${ }^{\#} P<0.05$ versus WT OGD neurons 


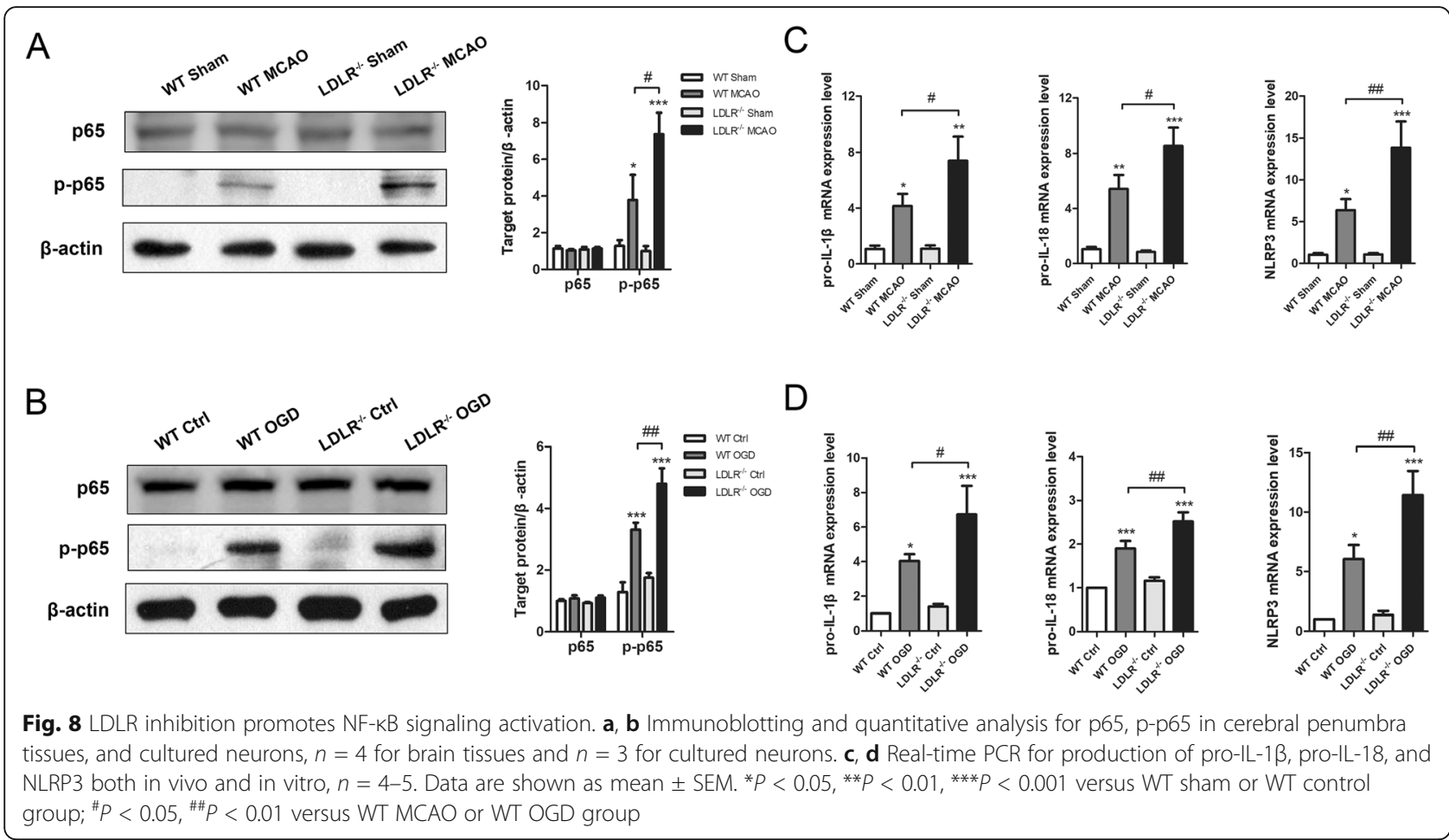

death in ischemic stroke [1, 32, 35, 36]. Recognition of DAMPs mediated by surface PPRs subsequently triggers enhanced downstream transcriptional activities of proIL-1 $\beta$, pro-IL-18, and NLRP3 via nuclear factor kappa B $(\mathrm{NF}-\mathrm{KB})$ in an autocrine or paracrine manner, which serves as signal 1 (priming) of the inflammatory response [34]. After NLRP3 binding with the adaptor protein ASC, pro-caspase- 1 is converted into its biologically active form and subsequently triggers the maturation of precursor IL-1 $\beta$ and IL-18 $[37,38]$. In addition, the NLRP3 inflammasome evokes proinflammatory GSDM D-executed pyroptotic cell death [39, 40]. As the key protein in pyroptosis, GSDMD belongs to a gasdermin family, including GSDMA, GSDMB, GSDMDC, GSDM D, GSDME, and DFNB59 [41, 42]. Evidence has shown that full-length GSDMD contains a $31-\mathrm{kDa}$ N-terminal fragment and a $22-\mathrm{kDa}$ C-terminal fragment, in which the $\mathrm{C}$-terminus exerts an auto-inhibitory function in the resting state [43]. Upon cleaved at the D276 cleavage site by caspase- 1 , the $\mathrm{N}$-terminus of GSDMD specifically anchors to cellular membrane lipids and oligomerizes to form permeability pores whose inner diameter is 10-14 $\mathrm{nm}[44,45]$. Thereafter, these pores dissipate cellular ionic gradients, which then cause water influx, cell swelling, eventual osmotic lysis, and release of inflammatory intracellular contents [12]. Pyroptosis has been demonstrated to occupy a crucial place in nerve cell death after cerebral ischemic injury [7, 10, 46]. Analogously, our study observed an upregulated expression of full-length
GSDMD and its N-terminus cleavage product via NF-kB signal-primed inflammasome cascades in the acute phase of cerebral ischemia. A similar increase of $\mathrm{N}$-terminal region oligomer-forming pores on the membranes of neurons was also revealed.

As an endocytic transmembrane receptor localized on the plasma membrane, LDLR can combine and take up extracellular ligands such as low-density lipoprotein and apolipoprotein E, playing a crucial regulatory role in lipid and cholesterol metabolism [47]. LDLR contains a cluster of seven ligand-binding repeats, an EGF homology region and a sugar domain in the extracellular domain, and an NPxY motif in the cytoplasmic domain mediating ligand endocytosis and signal transduction via coated pits [48]. The regulation of LDLR is implemented through sterol regulatory element binding proteins (SREBPs) and secreted proprotein convertase subtilisinlike/kexin type 9 (PCSK9) at the transcriptional and posttranscriptional levels, respectively [49]. It has been confirmed that chronic inflammation could disrupt the LDLR pathway, giving rise to lipid disorders in atherosclerosis, nonalcoholic fatty liver disease, diabetes, and chronic kidney disease $[14,50,51]$, while emerging studies have shown that LDLR in turn plays a vital role in inflammatory reactions. The overexpression of LDLR via reducing PCSK9 function was associated with a decreased inflammatory cytokine response and improved septic shock outcomes in both mice and humans [52]. Likewise, it was observed that PCSK9 fortified 


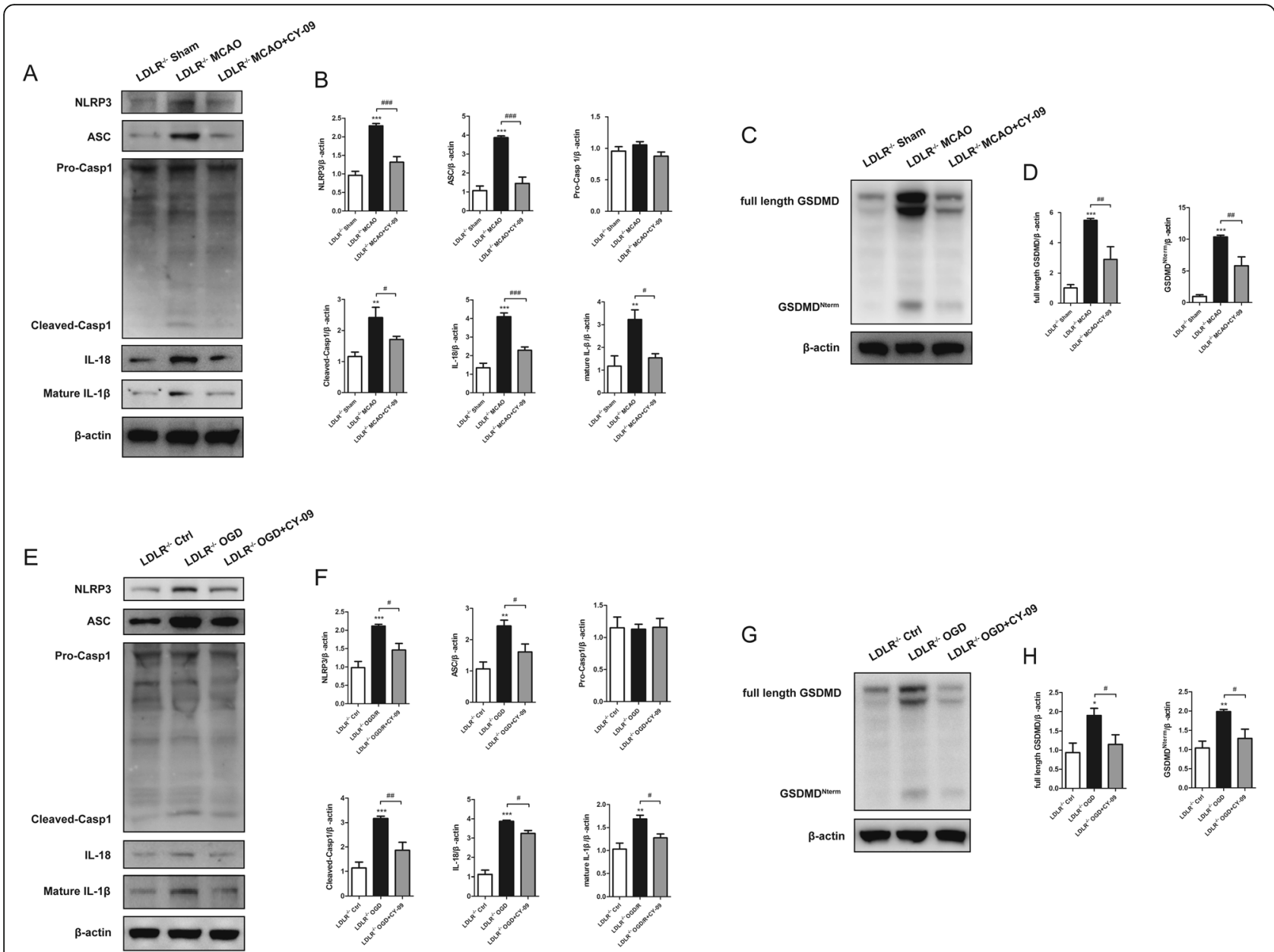

Fig. 9 NLRP3 suppression retards $L d$ lr deletion-induced augment of neuronal pyroptosis. $L d r^{-1-}$ MCAO mice and $L d r^{-1-}$ OGD neurons were treated with NLRP3 inhibitor, CY-09. a, b Expressions of NLRP3, ASC, caspase-1, IL-18, and mature IL-1 $\beta$ in peri-infarct region were analyzed by immunoblots $24 \mathrm{~h}$ after ischemia with or without CY-09 injection. c, d Western blotting and quantitative analysis for GSDMD. e, f Immunoblotting analysis and quantitation for NLRP3, ASC, caspase-1, IL-18, and mature IL-1 $\beta$ in $L d l^{-1-}$ neurons $24 \mathrm{~h}$ after OGD/R with or without CY-09 treatment. $\mathbf{g}, \mathbf{h}$ Immunoblots and quantitative analysis for GSDMD in cultured neurons after OGD. $n=4$ in each group. Data are shown as mean \pm SEM. ${ }^{*} P<0.05,{ }^{* *} P<0.01,{ }^{* * *} P<0.001$ versus $L d l r^{-1-}$

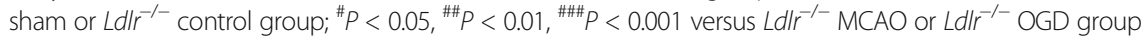

atherosclerotic inflammation and cell apoptosis in an LDLR-dependent mechanism [53]. In the brain, neuroinflammation of nerve cells was also shown to be mitigated in Ldlr transgenic mice with Alzheimer's disease [54], implying that LDLR may be involved in mediating the sterile inflammatory process of brain tissues. In this investigation, we found that $L d l r$ knockout pronouncedly exacerbated the risk of cerebral I/R-induced neuronal pyroptosis. The activation of the NLRP3 inflammasome in $L d l r^{-/}$mice was reinforced through the NF- $\mathrm{KB}$ signaling pathway following acute ischemia. In addition, inhibition of NLRP3 could reverse $L d l r$ deficiency-induced augment of neuronal inflammation and pyroptosis. These data indicated that LDLR could regulate NLRP3mediated neuronal pyroptosis after cerebral I/R, implying the protective role of LDLR as an inflammatory mediator in ischemic stroke. Consistent with our findings, a study has shown that Ldlr knockout augmented inflammatory cell infiltration in mouse aortas, although the underlying mechanism remains to be elucidated [55]. Nevertheless, our data conflict with a report that the increase in LDLR expression did not protect mice from LPS-induced death [56]. The contradictory roles of LDLR in the regulation of cell survival may be ascribed to the differences in cell types, experimental settings, and stimulus approaches.

Genetic ablation of $L d l r$ has been established to disrupt cholesterol homeostasis and cause the onset of atherosclerosis, such as familial hypercholesterolemia [16]. Ldlr deletion was verified to instigate the amplification of the inflammatory response in macrophages and other immune cells [57]. Hence, we assessed whether LDLR deletion would raise the level of cholesterol in brain tissue and consequently influences the 


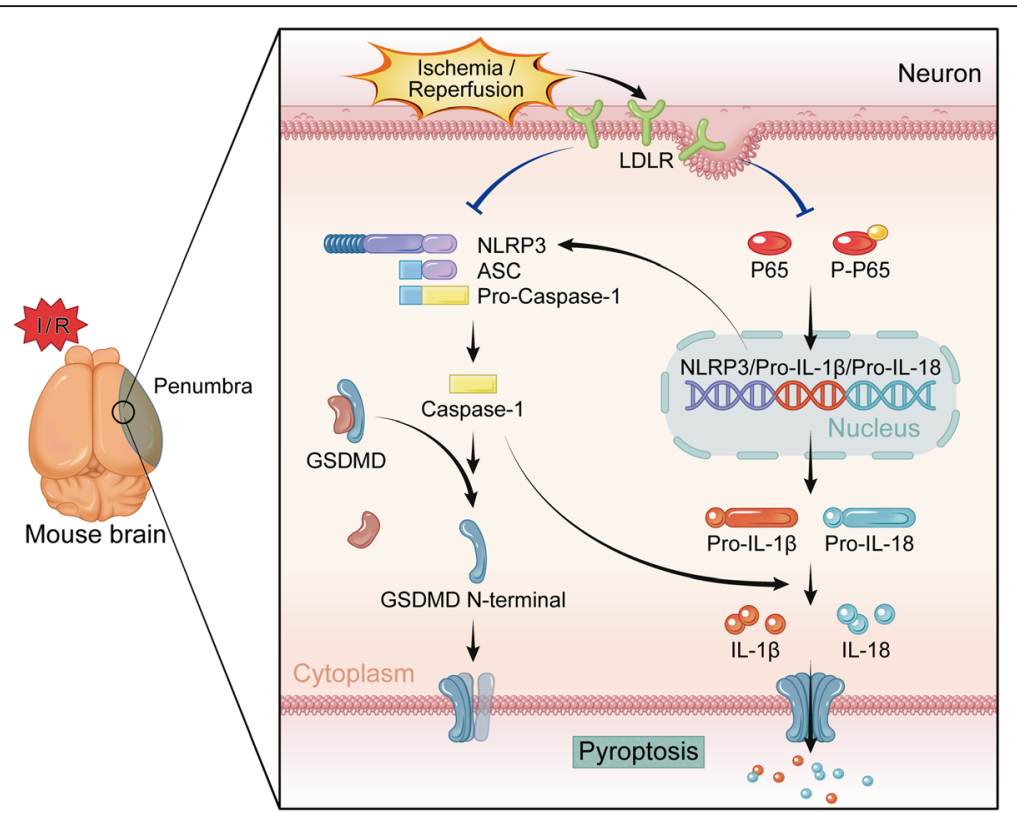

Fig. 10 Schematic diagram for the mechanisms of LDLR in the regulation of neuronal pyroptosis following cerebral ischemia. The expression of LDLR is downregulated after Cerebral I/R. LDLR restrains two-step processing pathways, including NF-KB signaling (priming) and NLRP3-ASCcaspase-1 inflammasome assembly, to mitigate generating inflammatory mediators and cytokines. The triggered caspase-1 cleaves GSDMD to promote the release of $\mathrm{N}$-terminal domain, which executes pores formation on neuronal membrane. The mature forms of IL-18 and IL-1 $\beta$ secreted through these pores are also alleviated, which facilitates anti-inflammatory effect post-stroke

inflammation motivation of nerve cells. Total cholesterol levels in the cortex, hippocampus, corpus callosum, and cerebellum were examined in our study, and the results showed no significant difference between WT and $\mathrm{Ldll}^{-1-}$ mice. Therefore, it may rule out the impact of cerebral cholesterol changes on inflammatory responses in the brain. It has been reported that mitochondria isolated from low-fat-fed $\mathrm{Ldlr}^{-1-}$ mice tissues produced more ROS, while attenuated LDLR degradation could cause a decrease in ROS generation [58, 59]. Another research also observed that $L d l r$ mutant macrophages presented increased levels of oxidants and inflammatory cytokines [60]. Simultaneously, experimental evidences demonstrated that the increase in ROS may serve as a triggering factor to activate NLRP3 inflammasome [6163]. Subsequent study has indicated that the elimination of ROS alleviated the cleavage of GSDMD and pyroptosis [64]; hence, further investigations are required to determine the potential mechanism of LDLR regulating ischemia-induced neuronal pyroptosis. Shc (p66) protein is a cellular signaling adaptor transducing signals of transmembrane proteins [65, 66]. Previous studies have demonstrated that Shc strongly promotes cellular ROS generation [67-69]. It is possible that LDLR may act on the ROS signaling pathway to regulate inflammasome activation downstream via interacting with the Shc protein.

\section{Conclusions}

In conclusion, this study demonstrated that LDLR regulated neuronal pyroptosis induced by cerebral I/R, which may exert a protective effect on neurons and improve neurological dysfunction following cerebral ischemia. To our knowledge, these results have for the first time disclosed the correlation of LDLR with NLRP3-induced neuronal pyroptosis post-stroke. Our findings highlighted a crucial role of LDLR in the suppression of neuroinflammation and may represent a therapeutic target in the treatment of inflammasome-associated diseases.

\section{Supplementary information}

Supplementary information accompanies this paper at https://doi.org/10. 1186/s12974-020-01988-X.

Additional file 1: Fig. S1. The location of LDLR protein in various brain regions. $(A, B)$ Double staining images of LDLR with NeuN were pictured in corpus callosum and hippocampus, $n=3$. Scale bar $=20 \mu \mathrm{m}$. CC, Corpus callosum.

Additional file 2: Fig. S2. Cholesterol levels in the plasma and different brain tissues of mice. (A) Cholesterol level in mice plasma. (B) Total cholesterol level in various brain areas. $n=4$ for all groups. Data are expressed as mean \pm SEM. ${ }^{* * *} P<0.001$ versus WT mice.

\section{Abbreviations}

ASC: Apoptosis-associated speck-like protein containing a caspase recruitment domain; Caspase-1: Cysteinyl aspartate specific proteinase-1;

DAMPs: Danger-associated molecular patterns; DAPI: 4',6-diamidino-2phenylindole; GSDMD: Gasdermin D; IL-1 $\beta$ : Interleukin-1 $\beta$; IL-18: Interleukin18; I/R: Ischemia/reperfusion; LDLR: Low-density lipoprotein receptor; 
MCAO: Middle cerebral artery occlusion; NLRP3: Nucleotide-binding oligomerization domain (Nod)-like receptor pyrin domain containing 3; OGD: Oxygen-glucose deprivation; PCSK9: Proprotein convertase subtilisin/ kexin type 9; PRR: Pattern recognition receptor; SREBPs: Sterol regulatory element binding proteins

\section{Acknowledgements}

Not applicable.

\section{Authors' contributions}

WZ and RS designed the experiments. RS, MP, and PX performed the experiment. RS and YX drafted or contributed to the writing of the manuscript. FH and QL analyzed the data. $\mathrm{JL}$ and $\mathrm{YH}$ contributed to the MCAO model. HG contributed to the cell culture. All authors read and approved the final manuscript.

\section{Funding}

This work was supported by the National Nature Science Foundation of China (no. 81671170) and Anhui Provincial Natural Science Foundation (no. 2008085QH368).

\section{Availability of data and materials}

All data generated or analyzed during this study are included in this published article and its supplementary files.

\section{Ethics approval and consent to participate}

All the experiments involving animals were approved by Experimental Animal Ethics Committee of Jinling Hospital. All experimental protocols were performed in accordance with the National Institutes of Health "Guide for the Care and Use of Laboratory Animals" (NIH Publications no. 8023, revised 2011).

\section{Consent for publication}

Not applicable.

\section{Competing interests}

All authors declare that they have no competing interests to disclosure.

\section{Author details}

${ }^{1}$ Department of Neurology, Jinling Clinical College of Nanjing Medical University, 305 East Zhongshan Road, Nanjing 210002, Jiangsu Province, China. ${ }^{2}$ Department of Neurology, Shanghai Changhai Hospital, Second Military Medical University/Naval Medical University, Shanghai 200433, China. ${ }^{3}$ Department of Neurology, Jinling Hospital, Medical School of Nanjing University, Nanjing 210002, China. ${ }^{4}$ Stroke Center \& Department of Neurology, The First Affiliated Hospital of USTC, Division of Life Sciences and Medicine, University of Science and Technology of China, Hefei 230036, Anhui, China. ${ }^{5}$ Department of Neurology, Jinling Hospital, the First School of Clinical Medicine, Southern Medical University, Nanjing 210002, China.

Received: 23 December 2019 Accepted: 7 October 2020

Published online: 05 November 2020

\section{References}

1. Yang F, Wang Z, Wei X, Han H, Meng X, Zhang Y, et al. NLRP3 deficiency ameliorates neurovascular damage in experimental ischemic stroke. J Cereb Blood Flow Metab. 2014;34(4):660-7.

2. Barrington J, Lemarchand E, Allan SM. A brain in flame; do inflammasomes and pyroptosis influence stroke pathology? Brain Pathol. 2017;27(2):205-12.

3. Hankey GJ. Stroke. Lancet. 2017;389(10069):641-54.

4. Liu S, Jin R, Xiao AY, Zhong W, Li G. Inhibition of CD147 improves oligodendrogenesis and promotes white matter integrity and functional recovery in mice after ischemic stroke. Brain Behav Immun. 2019;82:13-24.

5. Walsh JG, Muruve DA, Power C. Inflammasomes in the CNS. Nat Rev Neurosci. 2014;15(2):84-97.

6. Singh VRS, Veltkamp R, Liesz A. HMGB1 as a key mediator of immune mechanisms in ischemic stroke. Antioxid Redox Signal. 2016;24(12):635-51.

7. Poh L, Kang SW, Baik SH, Ng GYQ, She DT, Balaganapathy P, et al. Evidence that NLRC4 inflammasome mediates apoptotic and pyroptotic microglial death following ischemic stroke. Brain Behav Immun. 2019;75:34-47.
8. Malik A, Kanneganti TD. Inflammasome activation and assembly at a glance. J Cell Sci. 2017;130(23):3955-63.

9. Ding J, Wang K, Liu W, She Y, Sun Q, Shi J, et al. Pore-forming activity and structural autoinhibition of the gasdermin family. Nature. 2016; 535(7610):111-6.

10. Zhang D, Qian J, Zhang P, Li H, Shen H, Li X, et al. Gasdermin D serves as a key executioner of pyroptosis in experimental cerebral ischemia and reperfusion model both in vivo and in vitro. J Neurosci Res. 2019;97(6):645-60.

11. Wang Q, Wu J, Zeng Y, Chen K, Wang C, Yang S, et al. Pyroptosis: a proinflammatory type of cell death in cardiovascular disease. Clin Chim Acta. 2020:510:62-72.

12. Bergsbaken T, Fink SL, Cookson BT. Pyroptosis: host cell death and inflammation. Nat Rev Microbiol. 2009:7(2):99-109.

13. Zhang $P$, Cao L, Zhou $R$, Yang $X$, Wu M. The IncRNA Neat1 promotes activation of inflammasomes in macrophages. Nat Commun. 2019;10(1):1495.

14. Pieper-Fürst U, Lammert F. Low-density lipoprotein receptors in liver: old acquaintances and a newcomer. Biochim Biophys Acta. 2013;1831(7):1191-8.

15. Saher G, Stumpf SK. Cholesterol in myelin biogenesis and hypomyelinating disorders. Biochim Biophys Acta. 2015;1851(8):1083-94.

16. Go GW, Mani A. Low-density lipoprotein receptor (LdLr) family orchestrates cholesterol homeostasis. Yale J Biol Med. 2012;85(1):19-28.

17. Paciullo F, Fallarino F, Bianconi V, Mannarino MR, Sahebkar A, Pirro M. PCSK9 at the crossroad of cholesterol metabolism and immune function during infections. J Cell Physiol. 2017;232(9):2330-8.

18. Zheng J, Dai Q, Han K, Hong W, Jia D, Mo Y, et al. JNK-IN-8, a C-Jun Nterminal kinase inhibitor, improves functional recovery through suppressing neuroinflammation in ischemic stroke. J Cell Physiol. 2020;235(3):2792-9.

19. Yang $L$, Jiang $Y$, Wen $Z, X u X, X u X$, Zhu J, et al. Over-expressed EGR1 may exaggerate ischemic injury after experimental stroke by decreasing BDNF expression. Neuroscience. 2015:290:509-17.

20. Pan LL, Liang W, Ren Z, Li C, Chen Y, Niu W, et al. Cathelicidin-related antimicrobial peptide protects against ischaemia reperfusion-induced acute kidney injury in mice. Br J Pharmacol. 2020;177(12):2726-42.

21. Xu P, Liu Q, Xie Y, Shi X, Li Y, Peng M, et al. Breast cancer susceptibility protein 1 (BRCA1) rescues neurons from cerebral ischemia/reperfusion injury through NRF2-mediated antioxidant pathway. Redox Biol. 2018;18:158-72.

22. Xie Y, Guo H, Wang L, Xu L, Zhang X, Yu L, et al. Human albumin attenuates excessive innate immunity via inhibition of microglial Mincle/Syk signaling in subarachnoid hemorrhage. Brain Behav Immun. 2017;60:346-60.

23. Chen J, Li Y, Wang L, Zhang Z, Lu D, Lu M, et al. Therapeutic benefit of intravenous administration of bone marrow stromal cells after cerebral ischemia in rats. Stroke. 2001;32(4):1005-11.

24. D'Hooge R, De Deyn P. Applications of the Morris water maze in the study of learning and memory. Brain Res Brain Res Rev. 2001;36(1):60-90.

25. Wang R, Ma WG, Gao GD, Mao QX, Zheng J, Sun LZ, et al. Fluoro jade-C staining in the assessment of brain injury after deep hypothermia circulatory arrest. Brain Res. 2011;1372:127-32

26. Ye R, Yang Q, Kong X, Li N, Zhang Y, Han J, et al. Sevoflurane preconditioning improves mitochondrial function and long-term neurologic sequelae after transient cerebral ischemia: role of mitochondrial permeability transition. Crit Care Med. 2012:40(9):2685-93.

27. He WT, Wan H, Hu L, Chen P, Wang X, Huang Z, et al. Gasdermin D is an executor of pyroptosis and required for interleukin-1beta secretion. Cell Res. 2015;25(12):1285-98

28. Abulafia DP, de Rivero Vaccari JP, Lozano JD, Lotocki G, Keane RW, Dietrich WD. Inhibition of the inflammasome complex reduces the inflammatory response after thromboembolic stroke in mice. J Cereb Blood Flow Metab. 2009;29(3):534-44

29. Mascarenhas DPA, Cerqueira DM, Pereira MSF, Castanheira FVS, Fernandes TD, Manin GZ, et al. Inhibition of caspase-1 or gasdermin-D enable caspase8 activation in the Naip5/NLRC4/ASC inflammasome. PLoS Pathog. 2017; 13(8):e1006502

30. Mamantopoulos M, Ronchi F, Van Hauwermeiren F, Vieira-Silva S, Yilmaz $B$, Martens L, et al. Nlrp6- and ASC-dependent inflammasomes do not shape the commensal gut microbiota composition. Immunity. 2017; 47(2):339-348.e4.

31. Voet S, Srinivasan S, Lamkanfi M, van Loo G. Inflammasomes in neuroinflammatory and neurodegenerative diseases. EMBO Molecular Medicine. 2019;11(6):e10248.

32. Lammerding L, Slowik A, Johann S, Beyer C, Zendedel A. Poststroke inflammasome expression and regulation in the peri-infarct area by gonadal 
steroids after transient focal ischemia in the rat brain. Neuroendocrinology. 2016;103(5):460-75.

33. Cao G, Jiang $N$, Hu $Y$, Zhang $Y$, Wang $G$, Yin $M$, et al. Ruscogenin attenuates cerebral ischemia-induced blood-brain barrier dysfunction by suppressing TXNIP/NLRP3 inflammasome activation and the MAPK pathway. Int J Mol Sci. 2016;17(9):1418.

34. Fann DY, Lim YA, Cheng YL, Lok KZ, Chunduri P, Baik SH, et al. Evidence that NF-kappaB and MAPK signaling promotes NLRP inflammasome activation in neurons following ischemic stroke. Mol Neurobiol. 2018;55(2):1082-96.

35. Fann DY, Lee SY, Manzanero S, Tang SC, Gelderblom M, Chunduri P, et al. Intravenous immunoglobulin suppresses NLRP1 and NLRP3 inflammasomemediated neuronal death in ischemic stroke. Cell Death Dis. 2013:4e:790.

36. Ishrat T, Mohamed IN, Pillai B, Soliman S, Fouda AY, Ergul A, et al. Thioredoxin-interacting protein: a novel target for neuroprotection in experimental thromboembolic stroke in mice. Mol Neurobiol. 2015; 51(2):766-78.

37. Shichita T, Ito M, Yoshimura A. Post-ischemic inflammation regulates neural damage and protection. Front Cell Neurosci. 2014;8:319.

38. Kaushal V, Dye R, Pakavathkumar P, Foveau B, Flores J, Hyman B, et al. Neuronal NLRP1 inflammasome activation of caspase-1 coordinately regulates inflammatory interleukin-1-beta production and axonal degenerationassociated Caspase-6 activation. Cell Death Differ. 2015;22(10):1676-86.

39. Shi J, Gao W, Shao F. Pyroptosis: gasdermin-mediated programmed necrotic cell death. Trends Biochem Sci. 2017;42(4):245-54.

40. Gaidt MM, Hornung V. Pore formation by GSDMD is the effector mechanism of pyroptosis. EMBO J. 2016;35(20):2167-9.

41. Wang K, Sun Q, Zhong X, Zeng M, Zeng H, Shi X, et al. Structural mechanism for GSDMD targeting by autoprocessed caspases in pyroptosis. Cell. 2020;180(5):941-955.e920.

42. Li J, Zhao J, Xu M, Li M, Wang B, Qu X, et al. Blocking GSDMD processing in innate immune cells but not in hepatocytes protects hepatic ischemiareperfusion injury. Cell Death Dis. 2020;11(4):244.

43. Zhang L, Liu H, Jia L, Lyu J, Sun Y, Yu H, et al. Exosomes mediate hippocampal and cortical neuronal injury induced by hepatic ischemiareperfusion injury through activating pyroptosis in rats. Oxidative Med Cell Longev. 2019;2019:3753485.

44. Aglietti RA, Dueber EC. Recent insights into the molecular mechanisms underlying pyroptosis and gasdermin family functions. Trends Immunol. 2017;38(4):261-71.

45. Shi J, Zhao Y, Wang K, Shi X, Wang Y, Huang H, et al. Cleavage of GSDMD by inflammatory caspases determines pyroptotic cell death. Nature. 2015; 526(7575):660-5

46. Tu Y, Guo C, Song F, Huo Y, Geng Y, Guo M, et al. Mild hypothermia alleviates diabetes aggravated cerebral ischemic injury via activating autophagy and inhibiting pyroptosis. Brain Res Bull. 2019;150:1-12.

47. Willnow TE, Nykjaer A, Herz J. Lipoprotein receptors: new roles for ancient proteins. Nat Cell Biol. 1999;1(6):E157-62.

48. Li Y, Cam J, Bu G. Low-density lipoprotein receptor family: endocytosis and signal transduction. Mol Neurobiol. 2001;23(1):53-67.

49. Lagace TA. PCSK9 and LDLR degradation: regulatory mechanisms in circulation and in cells. Curr Opin Lipidol. 2014;25(5):387-93.

50. Liu J, Ma KL, Gao M, Wang CX, Ni J, Zhang Y, et al. Inflammation disrupts the $L D L$ receptor pathway and accelerates the progression of vascular calcification in ESRD patients. PLoS One. 2012;7(10):e47217.

51. Bombo RP, Afonso MS, Machado RM, Lavrador MS, Nunes VS, Quintao ER, et al. Dietary phytosterol does not accumulate in the arterial wall and prevents atherosclerosis of LDLr-KO mice. Atherosclerosis. 2013;231 (2):442-7.

52. Walley KR, Thain KR, Russell JA, Reilly MP, Meyer NJ, Ferguson JF, et al. PCSK9 is a critical regulator of the innate immune response and septic shock outcome. Sci Transl Med. 2014;6(258):258ra143.

53. Giunzioni I, Tavori H, Covarrubias R, Major AS, Ding L, Zhang Y, et al. Local effects of human PCSK9 on the atherosclerotic lesion. J Pathol. 2016;238(1):52-62

54. Kim J, Castellano JM, Jiang H, Basak JM, Parsadanian M, Pham V, et al. Overexpression of low-density lipoprotein receptor in the brain markedly inhibits amyloid deposition and increases extracellular a beta clearance. Neuron. 2009;64(5):632-44.

55. Ding Z, Liu S, Wang X, Khaidakov M, Dai Y, Mehta JL. Oxidant stress in mitochondrial DNA damage, autophagy and inflammation in atherosclerosis. Sci Rep. 2013;3:1077.
56. Berger JM, Loza Valdes A, Gromada J, Anderson N, Horton JD. Inhibition of PCSK9 does not improve lipopolysaccharide-induced mortality in mice. J Lipid Res. 2017;58(8):1661-9.

57. Tall AR, Yvan-Charvet L. Cholesterol, inflammation and innate immunity. Nat Rev Immunol. 2015;15(2):104-16.

58. Palee S, McSweeney CM, Maneechote C, Moisescu DM, Jaiwongkam T, Kerdphoo S, et al. PCSK9 inhibitor improves cardiac function and reduces infarct size in rats with ischaemia/reperfusion injury: benefits beyond lipidlowering effects. J Cell Mol Med. 2019;23(11):7310-9.

59. Paim BA, Velho JA, Castilho RF, Oliveira HC, Vercesi AE. Oxidative stress in hypercholesterolemic LDL (low-density lipoprotein) receptor knockout mice is associated with low content of mitochondrial NADP-linked substrates and is partially reversed by citrate replacement. Free Radic Biol Med. 2008;44(3):444-51.

60. Salerno AG, Rentz T, Dorighello GG, Marques AC, Lorza-Gil E, Wanschel A, et al. Lack of mitochondrial NADP(H)-transhydrogenase expression in macrophages exacerbates atherosclerosis in hypercholesterolemic mice. Biochem J. 2019:476(24):3769-89.

61. Abais JMXM, Zhang Y, Boini KM, Li PL. Redox regulation of NLRP3 inflammasomes: ROS as trigger or effector? Antioxid Redox Signal. 2015; 22(13):1111-29.

62. Minutoli L, Puzzolo D, Rinaldi M, Irrera N, Marini H, Arcoraci V, et al. ROSmediated NLRP3 inflammasome activation in brain, heart, kidney, and testis ischemia/reperfusion injury. Oxidative Med Cell Longev. 2016;2016:2183026.

63. Harijith A, Ebenezer DL, Natarajan V. Reactive oxygen species at the crossroads of inflammasome and inflammation. Front Physiol. 2014;5:352.

64. Wang Y, Shi P, Chen Q, Huang Z, Zou D, Zhang J, et al. Mitochondrial ROS promote macrophage pyroptosis by inducing GSDMD oxidation. J Mol Cell Biol. 2019;11(12):1069-82.

65. Wills MK, Jones N. Teaching an old dogma new tricks: twenty years of Shc adaptor signalling. Biochem J. 2012;447(1):1-16.

66. Sagi O, Budovsky A, Wolfson M, Fraifeld V. ShcC proteins: brain aging and beyond. Ageing Res Rev. 2015;19:34-42.

67. Costantino S, Paneni F, Mitchell K, Mohammed SA, Hussain S, Gkolfos C, et al. Hyperglycaemia-induced epigenetic changes drive persistent cardiac dysfunction via the adaptor p66 Shc. Int J Cardiol. 2018:268:179-86.

68. Kong X, Guan J, Li J, Wei J, Wang R. P66Shc-SIRT1 regulation of oxidative stress protects against cardio-cerebral vascular disease. Mol Neurobiol. 2016; 54(7):5277-85.

69. Francia P, Cosentino F, Schiavoni M, Huang Y, Perna E, Camici GG, et al. p66(Shc) protein, oxidative stress, and cardiovascular complications of diabetes: the missing link. J Mol Med (Berl). 2009;87(9):885-91.

\section{Publisher's Note}

Springer Nature remains neutral with regard to jurisdictional claims in published maps and institutional affiliations.

Ready to submit your research? Choose BMC and benefit from:

- fast, convenient online submission

- thorough peer review by experienced researchers in your field

- rapid publication on acceptance

- support for research data, including large and complex data types

- gold Open Access which fosters wider collaboration and increased citations

- maximum visibility for your research: over $100 \mathrm{M}$ website views per year

At $\mathrm{BMC}$, research is always in progress.

Learn more biomedcentral.com/submission 